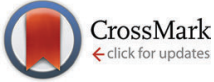

Cite this: New J. Chem., 2016 40,2028

Received (in Montpellier, France) 12th August 2015, Accepted 4th December 2015 DOI: $10.1039 / c 5 n j 02108 a$

www.rsc.org/njc

\title{
Aggregate-based sub-CMC solubilization of $n$-alkanes by monorhamnolipid biosurfactant
}

\author{
Hua Zhong, ${ }^{\text {abc }}$ Xin Yang, ${ }^{\text {ab }}$ Fei Tan, ${ }^{\text {ab }}$ Mark L. Brusseau, ${ }^{c}$ Lei Yang, ${ }^{\text {ab }}$ Zhifeng Liu, $^{\text {ab }}$ \\ Guangming Zeng ${ }^{\mathrm{ab}}$ and Xingzhong Yuan ${ }^{\mathrm{ab}}$
}

\begin{abstract}
Solubilization of $n$-decane, dodecane, tetradecane and hexadecane by monorhamnolipid biosurfactant (monoRL) at concentrations near the critical micelle concentration (CMC) was investigated. The apparent solubility of all four alkanes increases linearly with increasing monoRL concentration either below or above the CMC. The capacity of solubilization presented by the molar solubilization ratio (MSR), however, is stronger at monoRL concentrations below the CMC. The MSR decreases following the order dodecane > decane $>$ tetradecane $>$ hexadecane at monoRL concentrations below the CMC. Formation of aggregates at sub-CMC monoRL concentrations was demonstrated by dynamic light scattering (DLS) and cryo-transmission electron microscopy. DLS-based size $(d)$ and zeta potential of the aggregates decrease with increasing monoRL concentration. The surface excess $(\Gamma)$ of monoRL calculated based on alkane solubility and aggregate size data increases rapidly with increasing bulk monoRL concentration and then asymptotically approaches the maximum surface excess $\left(\Gamma_{\text {max }}\right)$. The relationship between $\Gamma$ and $d$ indicates that the excess of monoRL molecules at the aggregate surface greatly impacts the surface curvature. The results demonstrate formation of aggregates for alkane solubilization at monoRL concentrations below the CMC, indicating the potential of employing low concentrations of rhamnolipid for enhanced solubilization of hydrophobic organic compounds.
\end{abstract}

\section{Introduction}

Biosurfactants are amphiphilic molecules produced by microbes. They have the properties of typical surfactants such as lowering interfacial tension, wetting surfaces, foaming, and causing solubilization or emulsification of hydrophobic organic compounds (HOCs). Due to their advantages over synthetic surfactants, e.g. low toxicity, ${ }^{1}$ high degradability and environmental compatibility, ${ }^{1}$ and high efficiency, ${ }^{2,3}$ biosurfactants have received increased use for numerous applications in areas such as chemical manufacturing, pharmaceuticals, and contamination remediation. ${ }^{4}$ Solubilization of organic compounds is one of the key functions in the applications of biosurfactants. For example, biosurfactant-enhanced aquifer remediation for removal of non-aqueous-phase-liquid HOCs is primarily based on the mechanism of solubilization. ${ }^{5,6}$

\footnotetext{
${ }^{a}$ College of Environmental Science and Engineering, Hunan University, Changsha 410082, China.E-mail:yx2013@hnu.edu.cn,tanfei_2013@163.com, wlwyanglei@126.com,lzf18182002@163.com,zgming@hnu.edu.cn, yxz@hnu.edu.cn, zhonghua@hnu.edu.cn

${ }^{b}$ Key Laboratory of Environmental Biology and Pollution Control (Hunan University), Ministry of Education, Changsha, 410082, China ${ }^{c}$ Department of Soil, Water and Environmental Science, University of Arizona, Tucson, 1177 E $4^{\text {th }}$ St., Arizona 85721, USA. E-mail: zhonghua@email.arizona.edu, brusseau@email.arizona.edu
}

Solubilization of HOCs by surfactants has been studied extensively at high surfactant concentrations, i.e. higher than the critical micelle concentration (CMC). ${ }^{7-14}$ Micelles are considered to be of spherical shape with three zones for solubilization: the core, the corona, and the core-corona interface. ${ }^{15,16}$ It is typically assumed that solubilization enhancement of hydrophobic compounds only occurs at surfactant concentrations higher than CMC. ${ }^{11,16,17}$

The results of some studies, however, showed that surfactants also solubilize HOCs at sub-CMC concentrations. For example, results of our prior study showed that synthetic surfactants SDBS and Triton X-100 enhanced solubilization of hexadecane at concentrations below the CMC based on an aggregate formation mechanism. ${ }^{18}$ There is evidence of similar behavior for biosurfactants, with Zhang and Miller reporting that solubility of octadecane was enhanced by rhamnolipid biosurfactant at sub-CMC concentrations. It is interesting to note that the enhancement was much more significant at concentrations below the CMC than at concentrations above. ${ }^{8}$ It was assumed that this sub-CMC enhancement of octadecane solubilization was due to the decrease of water-octadecane interfacial tension. ${ }^{8}$ In our prior study of hexadecane solubilization by rhamnolipids, similar results were also observed. ${ }^{19}$ Research is needed to delineate the mechanisms contributing to the sub-CMC solubilization capability observed for biosurfactants. This information is also relevant for the commercial application of biosurfactants in terms of their cost-effectiveness. 

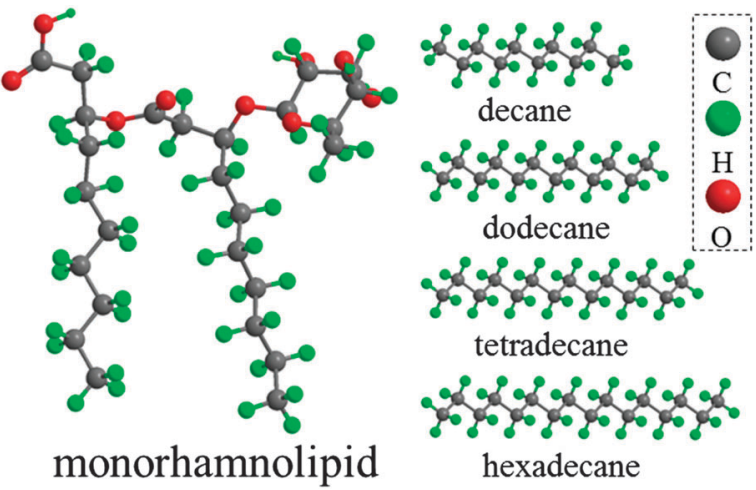

Fig. 1 Molecular structure of monoRL and the four $n$-alkanes.

To date, rhamnolipid is the most extensively studied biosurfactant and has the greatest application potential. Solubilization of $n$-alkanes by rhamnolipid at concentrations near the CMC was investigated in this study, with a focus on solubilization behavior at concentrations lower than of CMC. Monorhamnolipid (monoRL), a rhamnolipid with one rhamnose ring and two alkyl chains (Fig. 1), was used in this study. MonoRL is selected because it is the class of species that always exists in a rhamnolipid mixture and is the precursor for biosynthesis of dirhamnolipid. Results of our prior study also showed that it appears to have stronger ability over dirhamnolipid and synthetic surfactants to enhance HOC solubilization at low concentrations. ${ }^{3,18}$ It is considered an anionic surfactant under the experimental conditions in this study due to the carboxyl group in the molecule $\left(\mathrm{p} K_{\mathrm{a}}=5.6\right.$ under ambient temperature ${ }^{20}$ ). Four linear alkanes ( $n$-decane, $n$-dodecane, $n$-tetradecane and $n$-hexadecane) with different chain lengths were selected to represent HOCs. In addition to $n$-alkane solubility, characterization of alkanemonoRL aggregates, such as measurement of aggregate size and zeta potential and cryo-TEM-based observation of aggregate morphology, was undertaken. Finally, surfactant interface partition theory, an assumption of spherical aggregates, and surfactant mass balance was used to interpret the sub-CMC solubilization of the alkanes by the rhamnolipid.

\section{Theoretical background}

Based on the classical model for the structure of alkanesurfactant aggregates formed in a solution during alkane solubilization, the aggregates are assumed to be spherical, comprising alkane residing in the core zone and a layer of surfactant molecules at the surface with their alkyl chains intermingling with the alkane molecules in the core. Rhamnolipid molecules reside in bulk solution or in the outer layer of the aggregate, for which the partition can be described using Gibbs and Langmuir adsorption equations. ${ }^{21-23}$ In addition, the total mass of rhamnolipid in bulk solution and as aggregates is equal to the mass of rhamnolipid initially added. Based on these assumptions, partition of rhamnolipid between bulk solution and aggregate phase at solubilization equilibrium can be calculated using measures of interfacial tension and aggregate size. The theoretical details can be found in ref. 18 .

\section{Materials and methods}

\subsection{Materials}

The monoRL biosurfactant (purity $>99.9 \%$ ) was purchased from Zijin Biological Technology Co. Ltd (Huzhou, China). Constituent characterization of the monoRL is described by Zhong et al. ${ }^{24}$ The monoRL comprises five species of Rha- $\mathrm{C}_{10}-\mathrm{C}_{8}$, Rha- $\mathrm{C}_{10}-\mathrm{C}_{10: 1}$, Rha- $\mathrm{C}_{10}-\mathrm{C}_{10}$, Rha- $\mathrm{C}_{10}-\mathrm{C}_{12: 1}$ and Rha- $\mathrm{C}_{10}-\mathrm{C}_{12}$, where the abbreviation Rha- $\mathrm{C}_{x}-\mathrm{C}_{y: z}$ represents the individual component with $x$ and $y$ as the carbon atom number of each alkyl chain in the lipid moieties, and $z$ as the number of unsaturated bonds in the lipid moieties. Rha- $\mathrm{C}_{10}-\mathrm{C}_{10}$ is the major component with relative molar abundance of $75.5 \%$.

$n$-Alkanes (n-decane, $n$-dodecane, $n$-tetradecane and $n$-hexadecane) (purity $>99 \%)$ were purchased from SigmaAldrich (St. Louis, Mo., U.S.A.). The selected properties of $n$-alkanes are listed in Table 1 and molecule structures are shown in Fig. 1. $n$-Octane (purity $>95.0 \%$ ) and HPLC grade ethanol were purchased from Damao Chemical Reagent Co. Ltd (Tianjin, China). All other chemicals were of analytical grade and used as received. Ultra-pure water with electrical resistivity of $18.2 \mathrm{M} \Omega \mathrm{cm}$ produced by a UPT-II-40 (Ulupure, Chengdu, China) was used throughout the experiment. Phosphate buffer (PBS, $1.24 \mathrm{~g} \mathrm{~L}^{-1} \mathrm{KH}_{2} \mathrm{PO}_{4}$ and $1.35 \mathrm{~g} \mathrm{~L}^{-1} \mathrm{~K}_{2} \mathrm{HPO}_{4} \cdot 3 \mathrm{H}_{2} \mathrm{O}$, pH 6.8) was used as the background electrolyte solution for monoRL solubilization. It provides a stable concentration of counterions, which is important for application of the Gibbs adsorption equation for monoRL with an ionic nature. In this PBS buffer, the degree of dissociation for the monoRL is $94 \%$ based on $\mathrm{p} K_{\mathrm{a}}$ of 5.6. ${ }^{20}$ Such a high degree of dissociation also supports the

Table 1 Selected properties of $n$-alkanes and the alkane-PBS interfacial partitioning coefficients for monoRL

\begin{tabular}{|c|c|c|c|c|c|c|c|c|c|}
\hline n-Alkane & Formula & $\begin{array}{l}\text { Molecular weight } \\
\left(\mathrm{g} \mathrm{mol}^{-1}\right)\end{array}$ & $\begin{array}{l}\text { Water solubility }{ }^{a} \\
\left(\mu \mathrm{M}, 25^{\circ} \mathrm{C}\right)\end{array}$ & $\begin{array}{l}\log K_{\mathrm{ow}}{ }^{b} \\
\left(25^{\circ} \mathrm{C}\right)\end{array}$ & $\begin{array}{l}\text { Density }^{c} \\
\left(\mathrm{~g} \mathrm{~cm}^{-3}, 25{ }^{\circ} \mathrm{C}\right)\end{array}$ & $\begin{array}{l}\mathrm{CMC}^{d} \\
(\mu \mathrm{M})\end{array}$ & $\begin{array}{l}K \\
\left(\mathrm{~m}^{3} \mathrm{~mol}^{-1}\right)\end{array}$ & $\begin{array}{l}\Gamma_{\max } \\
\left(\mathrm{mol} \mathrm{m}^{-2}\right)\end{array}$ & $\begin{array}{l}A_{\mathrm{m}} \\
\left(\mathrm{nm}^{2}\right)\end{array}$ \\
\hline Decane & $\mathrm{C}_{10} \mathrm{H}_{22}$ & 142 & 0.37 & 5.01 & 0.73 & 150 & $0.98 \times 10^{3}$ & $3.1 \times 10^{-6}$ & 0.54 \\
\hline Dodecane & $\mathrm{C}_{12} \mathrm{H}_{26}$ & 170 & 0.02 & 6.10 & 0.75 & 155 & $1.81 \times 10^{3}$ & $2.9 \times 10^{-6}$ & 0.58 \\
\hline Tetradecane & $\mathrm{C}_{14} \mathrm{H}_{30}$ & 198 & 0.01 & 7.20 & 0.76 & 169 & $0.74 \times 10^{3}$ & $3.6 \times 10^{-6}$ & 0.46 \\
\hline Hexadecane & $\mathrm{C}_{16} \mathrm{H}_{34}$ & 226 & 0.0004 & 8.25 & 0.77 & 152 & $0.57 \times 10^{3}$ & $4.1 \times 10^{-6}$ & 0.41 \\
\hline
\end{tabular}

${ }^{a}$ Solubilities of $n$-alkanes are reported by NCBI (ref. 25-28). ${ }^{b}$ Octanol-water partition coefficient ( $\left.K_{\text {ow }}\right)$ values of $n$-alkanes from NCBI (ref. 25-28). ${ }^{c}$ Relative density (water $=1$ ) of $n$-alkanes from NCBI (ref. 25-28). ${ }^{d}$ Critical micelle concentration (CMC) for monoRL biosurfactant in the presence of $n$-alkanes obtained by $n$-alkane-PBS interfacial tension measurement (CMC obtained by surface tension measurement in the absence of $n$-alkanes is $166 \mu \mathrm{M})$. 
assumption in this study that the monoRL is anionic and resides only in the bulk solution or at the interface.

\subsection{Surface and interfacial tension measurement}

Interfacial tension between alkane and monoRL solutions with designated monoRL concentrations was measured at $30{ }^{\circ} \mathrm{C}$ with a tensiometer (JZ-200A, Chengde, China) using the Du Noüy Ring method. ${ }^{29}$ In brief, $15 \mathrm{~mL}$ of monoRL solution in PBS was prepared in a $50 \mathrm{~mL}$ glass beaker. $15 \mathrm{~mL}$ of alkane was then carefully added to the top of the monoRL solution without disturbing the solution. Before the interfacial tension was measured, the beaker was kept at $30{ }^{\circ} \mathrm{C}$ for $30 \mathrm{~min}$ to allow partitioning of monoRL to the water-alkane interface to reach equilibrium. The measurements were reproducible, with the differences in duplicate measurements within $\pm 0.2 \mathrm{mN} \mathrm{m}^{-1}$. For reference purposes, the surface tension (interfacial tension between air and solution) of the monoRL solution was also measured.

\subsection{Solubilization of $\boldsymbol{n}$-alkane by monoRL}

For each $n$-alkane:monoRL combination, $50 \mu \mathrm{L}$ of alkane was pipetted and spread on the bottom of a $25 \mathrm{~mL}$ glass flask. $10 \mathrm{~mL}$ of monoRL solution in PBS was then added to the flask and incubated on a reciprocal shaker at $30^{\circ} \mathrm{C}, 120 \mathrm{rpm}$ for $24 \mathrm{~h}$ to allow solubilization to reach equilibrium (the result of a preliminary test showed that alkane solubility did not change after $24 \mathrm{~h}$ ). The flasks were allowed to stand for $2 \mathrm{~h}$ for phases to separate, then $4 \mathrm{~mL}$ of aqueous solution saturated with only pseudo-solubilized alkane was separated using the method described by Zhong et al. ${ }^{19} 1 \mathrm{~mL}$ of the collected sample was removed for alkane concentration measurement, and another $2 \mathrm{~mL}$ was used for measurement of size and zeta potential of the aggregates. The alkane concentration was measured using gas chromatography (Agilent GC 6890N) following the procedures described by Zhong et al. ${ }^{19}$ A control containing $10 \mathrm{~mL}$ monoRL solution and no alkane was used to quantify loss of monoRL due to adsorption to the inner walls of the flask.

The size and zeta potential of aggregate particles were measured using a ZEN3600 Zetasizer Nano (Malvern Instruments, U.K.). The particle size was determined based on the method of dynamic light scattering (DLS) at $633 \mathrm{~nm}$ with a He-Ne laser working at $4 \mathrm{mV}$ power. $1 \mathrm{~mL}$ of sample was loaded to the DTS-0012 cell and maintained at $30{ }^{\circ} \mathrm{C}$. The scattered light was collected by the receptor at an angle of $173^{\circ}$ from the light path. The size of the aggregates was expressed in terms of hydrodynamic diameter, which was calculated using the software associated with the instrument. To obtain the zeta potential of the aggregates, approximately $1 \mathrm{~mL}$ of sample was loaded in the DTS1060 folded capillary cell and the electrophoretic mobility of the aggregate particles was measured at $30{ }^{\circ} \mathrm{C}$ under automatic voltage using laser Doppler velocimetry with the M3-PALS technique to avoid electroosmosis. The measured data were converted into the corresponding zeta potential by applying the Helmholtz-Smoluchowski equation. $^{30}$

\subsection{Cryo-Transmission Electron Microscopy (cryo-TEM) observation of hexadecane-monoRL aggregates}

A $4 \mu \mathrm{L}$ drop of solubilized hexadecane solution was placed on a copper grid and then sent to a FEI Vitrobot sample plunger. The excess sample was removed with a filter paper. The grid was then immediately plunged into a bath of liquid ethane and transferred to a bath of liquid nitrogen. The samples were stored in a GATAN model cryo-transfer unit in liquid nitrogen. The morphology of surfactant-hexadecane aggregates was viewed with a Tecnai F20 cryo-transmission electron microscope (FEI, Hillsboro, Oregon) at $120 \mathrm{kV}$.

\section{Results and discussion}

\subsection{CMC and interfacial partitioning parameters}

The dependence of air-PBS and $n$-alkane-PBS interfacial tension on monoRL concentration is presented in Fig. 2a. CMCs calculated using the method described by Zhong et al. ${ }^{31}$ are presented in Table 1. The CMCs of monoRL obtained using surface tension and interfacial tension measurements are similar to one another, showing that the non-aqueous phase
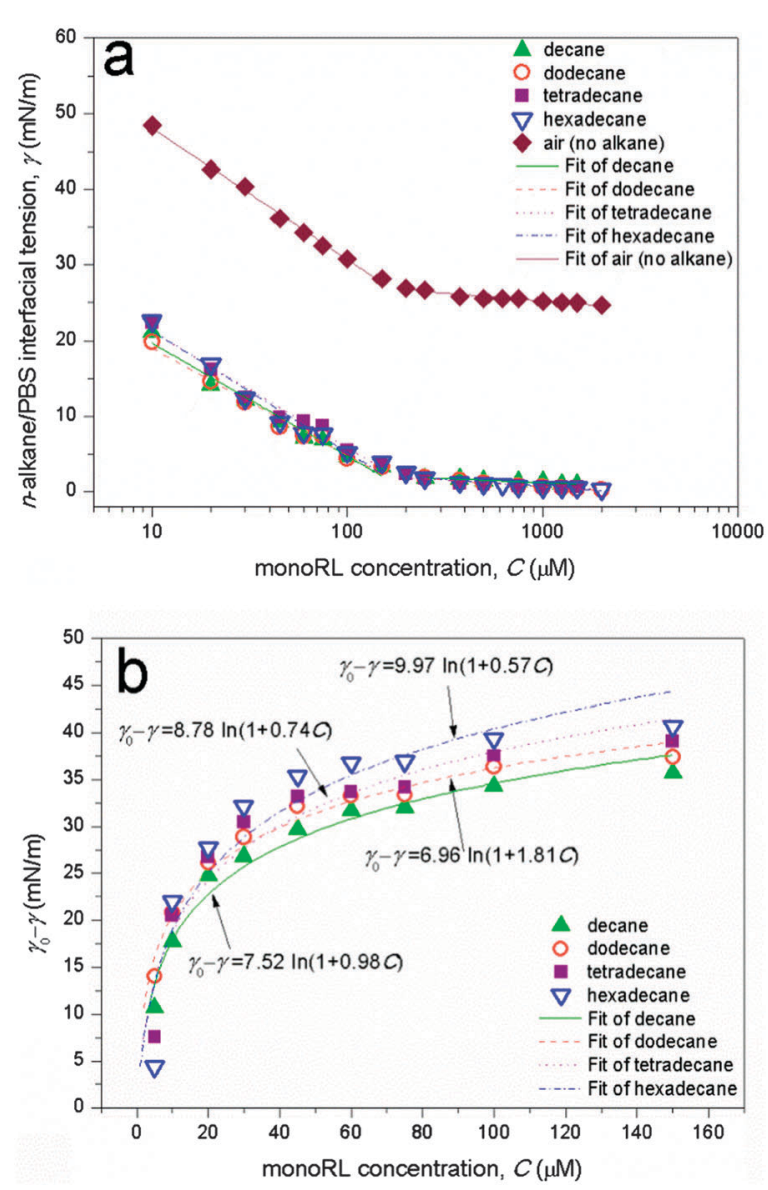

Fig. 2 (a) The air-PBS and $n$-alkane-PBS interfacial tension as a function of monoRL concentration. (b) Interfacial tension-concentration relation regression at monoRL concentrations below CMC using the Szyszkowski equation (eqn (3) in ref. 18). 
(air or $n$-alkane) has little impact on the CMC. An average CMC of $158 \pm 9 \mu \mathrm{M}$ is obtained.

The interfacial tension data at sub-CMC monoRL concentration was well fit by eqn (3) in ref. 18 (Fig. 2b). The Langmuir adsorption constant $(K)$, maximum interfacial access $\left(\Gamma_{\max }\right)$, and minimum area per molecule $\left(A_{\mathrm{m}}\right)$ obtained for the adsorption are summarized in Table $1 . K$ decreases following the order dodecane $>$ decane $>$ tetradecane $>$ hexadecane. Alkyl chain lengths in the monoRL are similar to those of dodecane and decane (Fig. 1), which may be favorable for hydrophobic interaction between monoRL and alkane molecules at the interface and hence lower the Gibbs energy, resulting in a stronger partitioning of monoRL at the interface in the cases of dodecane and decane. However, $\Gamma_{\max }$ is large $\left(A_{\mathrm{m}}\right.$ is small) for tetradecane and hexadecane, showing that when the adsorption is saturated the monoRL molecules are more compacted at the interface for long-chain alkanes.

\subsection{Solubilization of $n$-alkanes by monoRL}

Apparent solubility of alkanes as a function of total monoRL concentration, $C_{0}$, is shown in Fig. 3. For all four alkanes, the solubility is enhanced at monoRL concentrations below the CMC. The apparent solubility of each alkane increased linearly
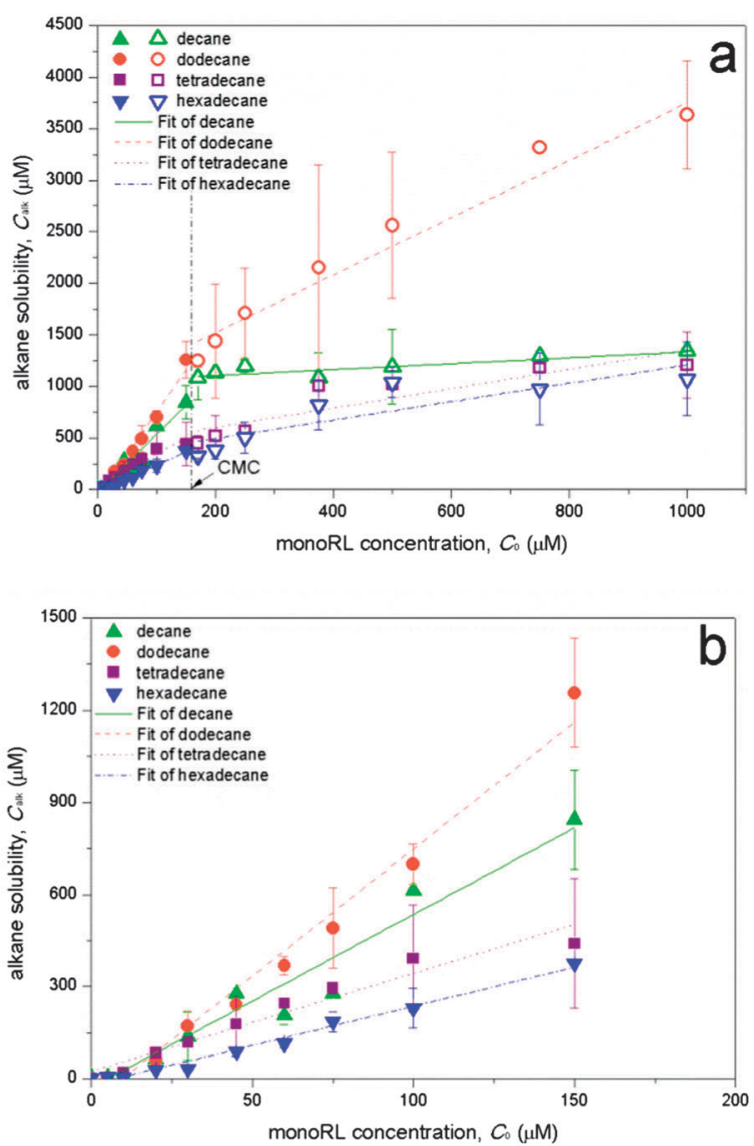

Fig. 3 (a) Apparent $n$-alkane solubility $\left(C_{\text {alk }}\right)$ versus monoRL total concentration $\left(C_{0}\right)$. Two sets of regressions represent data below and above the CMC. (b) Expanded plot of $C_{\text {alk }}-C_{0}$ relation for $C_{0}$ lower than of the CMC. Error bars show mean \pm standard deviation.
Table 2 The molar solubilization ratio (MSR) for alkane solubilization by monoRL

\begin{tabular}{lll}
\hline & \multicolumn{2}{l}{ MSR } \\
\cline { 2 - 3 }$n$-Alkane & Below CMC & Above CMC \\
\hline Decane & 5.73 & 0.29 \\
Dodecane & 8.28 & 2.91 \\
Tetradecane & 3.27 & 0.94 \\
Hexadecane & 2.55 & 0.89 \\
\hline
\end{tabular}

with monoRL concentration at different rates below and above the CMC.

The solubilization capacity of a surfactant for a HOC is presented by the molar solubilization ratio (MSR), which is defined as the increase of solubilized HOC concentration $\left(\mathrm{mol} \mathrm{L}^{-1}\right)$ per unit increase of surfactant concentration $\left(\mathrm{mol} \mathrm{L}^{-1}\right)$ in the solution or the slope of the linear solubilization curve. $^{32}$ The MSR for the four alkanes are listed in Table 2. MSR for all four alkanes are significantly higher at monoRL concentrations below the CMC than above the CMC. Similar results were observed for octadecane solubilization by monoRL ${ }^{8}$ and hexadecane solubilization by SDBS (also an anionic surfactant). ${ }^{18}$

These observations indicate a difference in the modes of alkane solubilization below and above the CMC. The MSR decreases in the order dodecane $>$ decane $>$ tetradecane $>$ hexadecane at monoRL concentrations below the CMC (Table 2), which is the same as the order for $K$. This indicates a relationship between alkane solubilization and interfacial partitioning of monoRL. It can be noted that the MSR for hexadecane solubilization by the monoRL at sub-CMC concentrations (2.55) is larger than that for SDBS (0.84) and Triton $\mathrm{X}-100$ (1.90), ${ }^{18}$ indicating higher solubilization efficiency of the biosurfactant monoRL over synthetic surfactants. This is probably due to the presence of two alkyl chains in the monoRL molecule.

\subsection{Size and zeta potential of aggregates}

The formation of aggregates was detected by aggregate size measurement using the DLS method. A single peak is observed for the number-based particle size distribution profile, indicating formation of one consistent size of aggregate (Fig. 4). The aggregates were observed directly with cryo-TEM, and the spherical aggregate morphology is confirmed (Fig. 5). Moreover, the size of the aggregates measured by cryo-TEM is similar to the measured size from DLS. The aggregates shown by cryo-TEM do not appear when hexadecane is equilibrated with an aqueous solution containing no monoRL (data not shown).

For all four alkanes, the DLS particle size first decreases rapidly with increase of $C_{0}$, and then stabilizes with increase of $C_{0}$ above the CMC (Fig. 6). By amongst the alkanes, it is observed that the aggregates size at monoRL concentration equivalent to the CMC decreases in the order decane $\approx$ dodecane $>$ tetradecane $>$ hexadecane. This order is in contrast to the order of $\Gamma_{\max }$ for these four alkanes, which increases in the order decane $\approx$ dodecane $<$ tetradecane $<$ hexadecane (Table 1). 


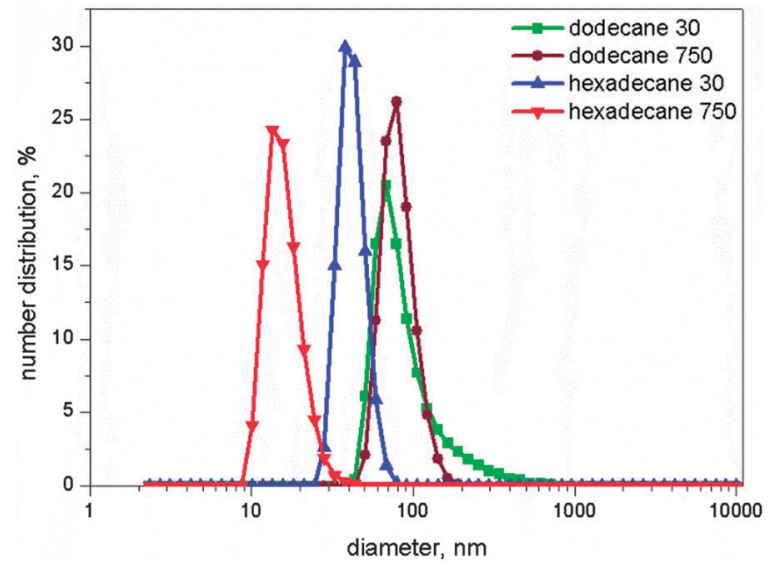

Fig. 4 Number distribution of aggregate particles for solubilization of dodecane and hexadecane by monoRL at concentrations of $30 \mu \mathrm{M}$ and $750 \mu \mathrm{M}$.
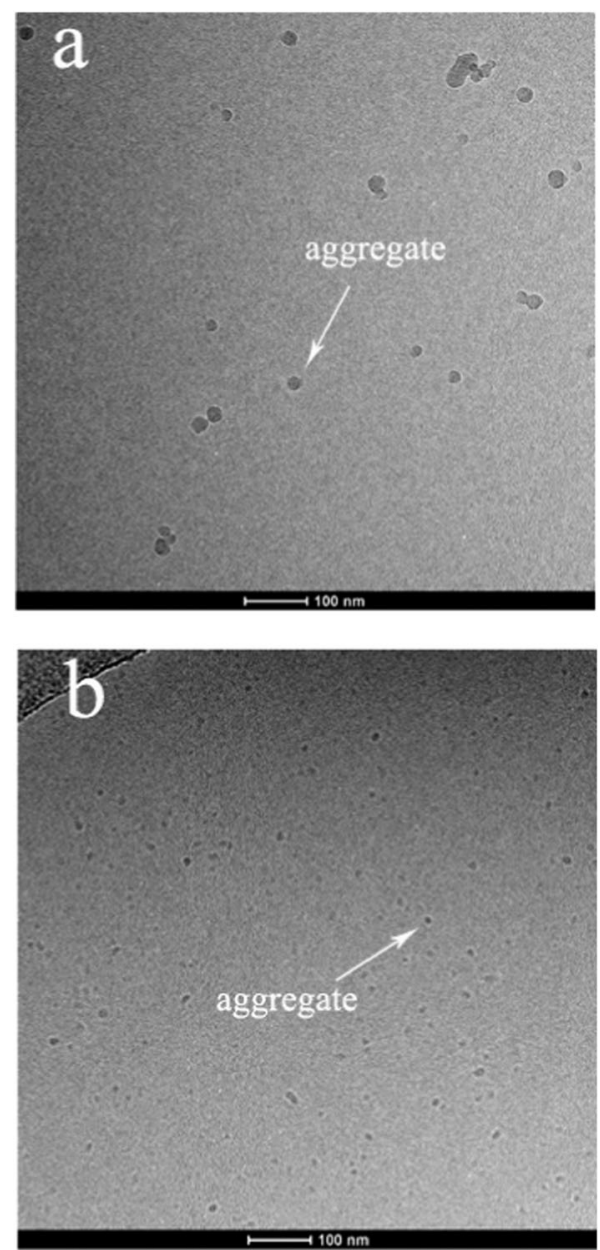

Fig. 5 Cryogenic-transmission electron microscopy (cryo-TEM) images showing aggregates for the solubilization of hexadecane by monoRL at monoRL concentrations of (a) $30 \mu \mathrm{M}$ (below CMC) and (b) $750 \mu \mathrm{M}$ (above CMC).

Zeta potentials of the aggregates are shown in Fig. 7. The aggregates are negatively charged. The change of zeta potential with increase of $C_{0}$ exhibits a similar trend for all

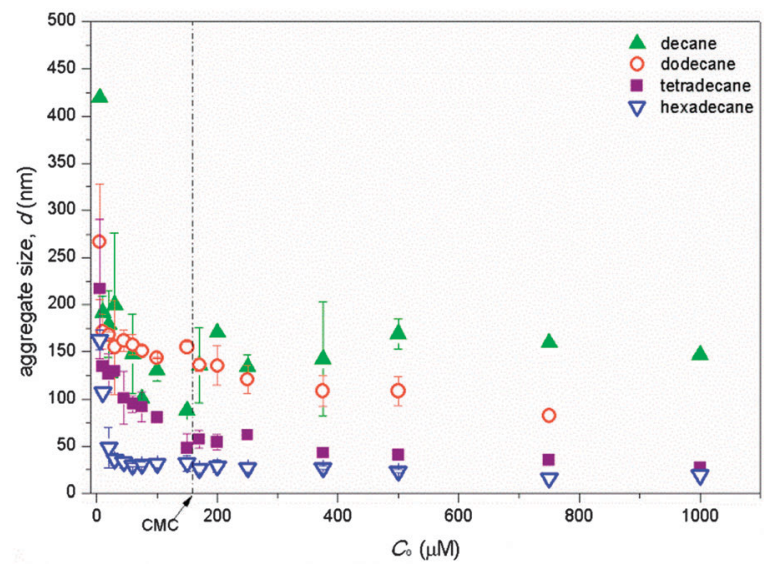

Fig. 6 DLS aggregate size (diameter, $d$ ) versus the total monoRL concentration $\left(C_{0}\right)$ for $n$-alkane solubilization. Error bars show mean \pm standard deviation.

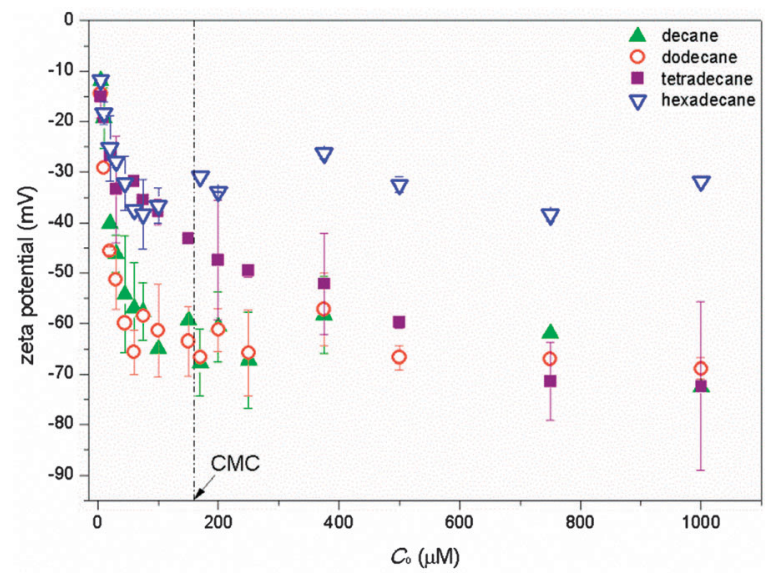

Fig. 7 Zeta potential of aggregates versus the monoRL total concentration $\left(C_{0}\right)$ for $n$-alkane solubilization. Error bars show mean \pm standard deviation.

four alkanes. It decreases rapidly with increase of $C_{0}$ to the CMC, and then stabilizes or decreases slowly with further increase of monoRL concentration.

\subsection{Partitioning of monoRL and its relation with aggregation}

No emulsion of alkanes in the presence of monoRL was observed in the experiments. Adsorption of monoRL to the inner walls of the flask was minimal (data not shown). Because a very limited volume of alkane ( $50 \mu \mathrm{L}$, see Materials and methods section) was used, partitioning of monoRL to the alkane phase, or to the interface between the floating mass of alkane and the aqueous phase (less than $1 \mathrm{~cm}^{2}$ in contrast to the magnitude of $10-10^{3} \mathrm{~cm}^{2}$ for the total surface area of the aggregates according to calculation below), was minimal. Therefore, the monoRL can be assumed to reside either in the bulk aqueous solution or in the aggregates. Due to the extremely low water solubility and high octanol-water partition coefficient $\left(K_{\mathrm{ow}}\right)$ of these four alkanes (Table 1), the amount of freely-dissolved alkane in the bulk 
aqueous phase is minimal and all the solubilized alkane is assumed to be associated with aggregates. Therefore, based on the spherical aggregate assumption, the aggregate surface excess, $\Gamma$, and the bulk concentration, $C_{\mathrm{w}}$, of monoRL monomer were calculated by applying eqn (2) and (5) in ref. 18 using $\Gamma_{\max }$ and $K$ previously obtained.

For all four alkanes, a linear relationship between the apparent solubility of alkane, $C_{\text {alk }}$, and $C_{\mathrm{w}}$ is observed with increase of $C_{\mathrm{w}}$ to the CMC (Fig. 8a). This is similar to the relationship between $C_{\text {alk }}$ and $C_{0}$ (the total monoRL concentration in solution) (Fig. 3). By comparing the slopes of the $C_{\text {alk }}-C_{0}$ profiles at $C_{0}$ below the CMC with those of the $C_{\text {alk }}-C_{\mathrm{w}}$ profiles (5.7 versus 7.5 for decane, 8.3 versus 10.8 for dodecane, 3.3 versus 5.3 for tetradecane, and 2.55 versus 6.3 for hexadecane), the percentage of the aggregate-associated monoRL is calculated to be $24 \%, 23 \%, 38 \%$, and $59 \%$ of the total for decane, dodecane, tetradecane, and hexadecane, respectively. Note that the aggregate size for hexadecane is significantly smaller than that for the other three alkanes at $C_{0}$ lower than CMC. The higher surface area for smaller particles is responsible for the enhanced partition of monoRL to the aggregates, in spite of the fact that the $K$ and $C_{\text {alk }}$ for hexadecane are the smallest among the four alkanes.
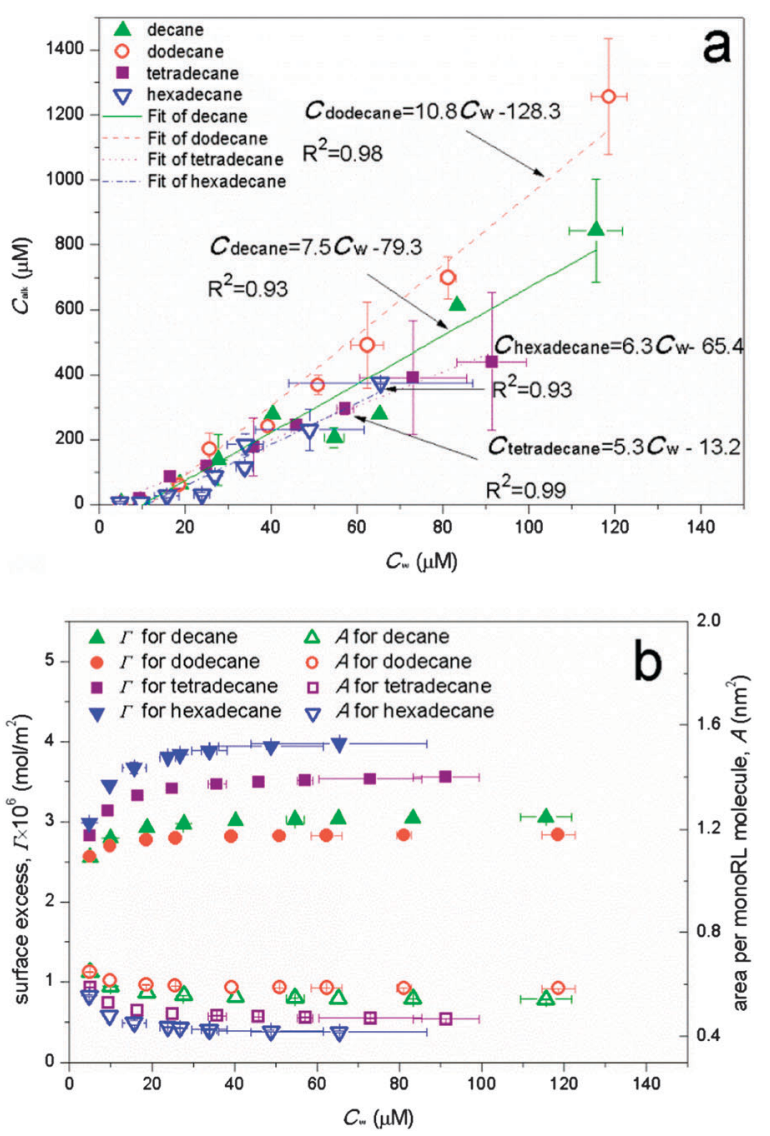

Fig. 8 (a) Apparent solubility of $n$-alkane $\left(C_{\text {alk }}\right)$ versus the monoRL bulk concentration $\left(C_{\mathrm{w}}\right)$ at $C_{\mathrm{w}}$ below CMC. (b) Surface excess $(\Gamma)$ and molecule area $(A)$ of monoRL on the aggregate surfaces versus monoRL bulk concentration $\left(C_{\mathrm{w}}\right)$. Error bars show mean \pm standard deviation.

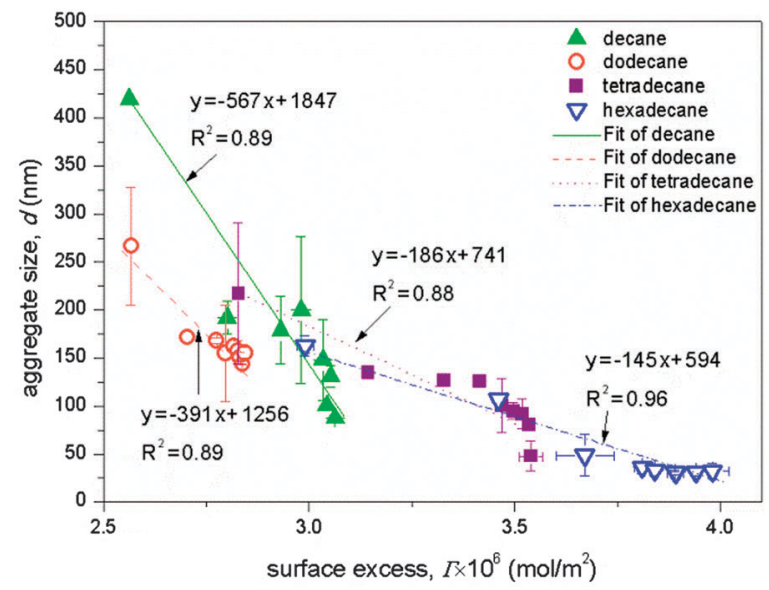

Fig. 9 Aggregate diameters $(d)$ versus surface excess of monoRL $(\Gamma)$ at monoRL bulk concentration $\left(C_{w}\right)$ below the CMC. Error bars show mean \pm standard deviation.

The dependence of monoRL surface excess $(\Gamma)$ and molecule area $(A)$ versus $C_{\mathrm{w}}$ are presented in Fig. 8b. A rapid increase of $\Gamma$ and decrease of $A$ with increasing $C_{\mathrm{w}}$ are observed when $C_{\mathrm{w}}$ is low. Further increase of $C_{\mathrm{w}}$ causes asymptotic approach of $\Gamma$ and $A$ to $\Gamma_{\max }$ and $A_{\mathrm{m}}$, respectively. More significant change of $\Gamma$ and $A$ is observed for the long-chain alkanes (tetradecane and hexadecane). Based on eqn (2) in ref. $18, \Gamma$ is more sensitive to change of $C_{\mathrm{w}}$ with a smaller $K$. $K$ for the four alkanes follows the order dodecane $>$ decane $>$ tetradecane $>$ hexadecane (Table 1). Thus, the most significant change of $\Gamma$ and $A$ over the broadest range of $C_{\mathrm{w}}$ occurred for hexadecane.

As shown in Fig. 9, for all four alkanes, aggregate size, $d$, decreases with the increase of monoRL surface excess in the aggregates, such that $d$ approaches the stabilized minimum aggregate size $\left(d_{\min }\right)$ as $\Gamma$ approaches $\Gamma_{\max }$. This result indicates that the curvature of the aggregate surface increases with increasing surface excess of monoRL molecules. Because monoRL is anionic and $94 \%$ of the monoRL molecules dissociate in PBS, the presence of monoRL causes a negative aggregate surface charge. Enhancement in electrostatic repulsion induces unequal rate of approach for polar and hydrophobic moieties between molecules, and therefore an increase in aggregate surface curvature (Fig. 10). Thus, the aggregate size, $d$, decreases with increasing $\Gamma$. Zeta potential is a function of both

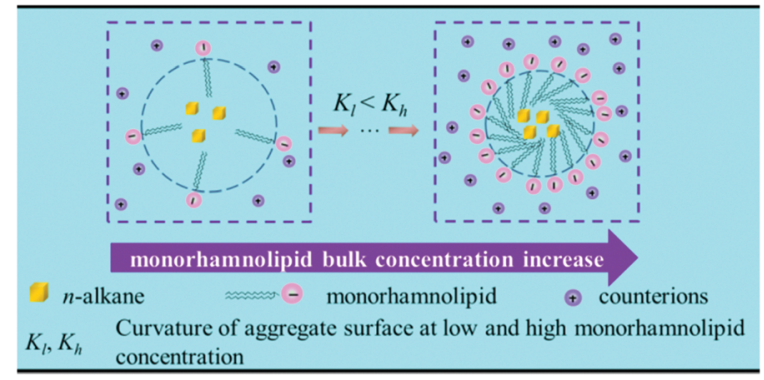

Fig. 10 Schematic diagram of alkane-monoRL aggregate formation at monoRL concentrations below the CMC. 
particle size and surface charge density. ${ }^{30,33,34}$ Therefore, it is essentially a function of $\Gamma$ and its change also exhibits an asymptotic pattern of decrease at concentrations lower than the CMC (see Fig. 7).

When monoRL concentration in a bulk solution $\left(C_{\mathrm{w}}\right)$ is higher than the CMC, $\Gamma$ at the aggregate surface reaches $\Gamma_{\max }$ and the size of aggregates reaches the minimum, giving low efficiency for alkane solubilization. As a result, the MSR at monoRL concentrations above the CMC is significantly smaller than that for monoRL concentrations below the CMC.

\section{Conclusion}

The results of this study demonstrated that monoRL biosurfactant at concentrations lower than the critical micelle concentration can enhance $n$-alkane solubilization. The results also support the fact that such solubilization enhancement is caused by an aggregate formation mechanism. Moreover, the solubilization enhancement at sub-CMC concentrations is more significant for the alkanes with chain lengths similar to the monoRL alkyl chain lengths. This appears to be the first report delineating the mechanism responsible for the sub-critical micelle concentration solubilization of hydrophobic organic compounds by a biosurfactant, which successfully explains observations of sub-CMC solubilization of alkanes by rhamnolipid in prior studies (i.e. ref. 8 and 19). The study is of importance to promote a better understanding of the solubilization behavior of hydrophobic organic compounds by rhamnolipid and for economical application of rhamnolipid biosurfactants in related areas. Future studies should aim to determine sub-critical micelle concentration solubilization behavior of rhamnolipid for other classes of hydrophobic organic compounds and in other matrices such as porous media.

\section{Acknowledgements}

The authors thank the Center for Integrative Imaging (CII) at the University of Science and Technology of China for cryo-TEM analysis. This study was funded by the National Natural Science Foundation of China (51378192, 51378190, 51308200 and 21276269) and the Program for Changjiang Scholars and Innovative Research Team in University (IRT-13R17). Additional support was provided by the NIEHS Superfund Research Program (P42 ES04940).

\section{References}

1 H. Y. Fu, G. M. Zeng, H. Zhong, X. Z. Yuan and G. H. Huang, Biodegradation, 2007, 18, 303-310.

2 X. L. Liu, G. M. Zeng, L. Tang, H. Zhong, R. Y. Wang, H. Y. Fu, Z. F. Liu, H. L. Huang and J. C. Zhang, Process Biochem., 2008, 43, 1300-1303.

3 H. Zhong, Y. Liu, Z. F. Liu, Y. B. Jiang, F. Tan, G. M. Zeng, X. Z. Yuan, M. Yan, Q. Y. Niu and Y. S. Liang, Int. Biodeterior. Biodegrad., 2014, 94, 152-159.
4 I. M. Banat, A. Franzetti, I. Gandolfi, G. Bestetti, M. G. Martinotti, L. Fracchia, T. J. Smyth and R. Marchant, Appl. Microbiol. Biotechnol., 2010, 87, 427-444.

5 L. M. Abriola, C. D. Drummond, E. J. Hahn, K. F. Hayes, T. C. G. Kibbey, L. D. Lemke, K. D. Pennell, E. A. Petrovskis, C. A. Ramsburg and K. M. Rathfelder, Environ. Sci. Technol., 2005, 39, 1778-1790.

6 J. Childs, E. Acosta, M. D. Annable, M. C. Brooks, C. G. Enfield, J. H. Harwell, M. Hasegawa, R. C. Knox, P. S. Rao, D. A. Sabatini, B. Shiau, E. Szekeres and A. L. Wood, J. Contam. Hydrol., 2006, 82, 1-22.

7 D. E. Kile and C. T. Chiou, Environ. Sci. Technol., 1989, 23, 832-838.

8 Y. Zhang and R. M. Miller, Appl. Environ. Microbiol., 1992, 58, 3276-3282.

9 K. D. Pennell, L. M. Abriola and W. J. Weber Jr, Environ. Sci. Technol., 1993, 27, 2332-2340.

10 J. E. McCray, G. Bai, R. M. Maier and M. L. Brusseau, J. Contam. Hydrol., 2001, 48, 45-68.

11 J. S. Clifford, M. A. Ioannidis and R. L. Legge, J. Colloid Interface Sci., 2007, 305, 361-365.

12 J. D. Albino and I. M. Nambi, J. Environ. Sci. Health, Part A: Toxic/Hazard. Subst. Environ. Eng., 2009, 44, 1565-1573.

13 S. Damrongsiri, C. Tongcumpou, P. Weschayanwiwat and D. A. Sabatini, J. Hazard. Mater., 2010, 181, 1109-1114.

14 K. Matsuoka, R. Yamashita, M. Ichinose, M. Kondo and T. Yoshimura, Colloids Surf., A, 2014, 456, 83-91.

15 A. Patist, J. R. Kanicky, P. K. Shukla and D. O. Shah, J. Colloid Interface Sci., 2002, 245, 1-15.

16 M. A. Mir, O. A. Chat, M. H. Najar, M. Younis, A. A. Dar and G. M. Rather, J. Colloid Interface Sci., 2011, 364, 163-169.

17 A. R. Tehrani-Bagha and K. Holmberg, Materials, 2013, 6, 580-608.

18 H. Zhong, L. Yang, G. Zeng, M. L. Brusseau, Y. Wang, Y. Li, Z. Liu, X. Yuan and F. Tan, RSC Adv., 2015, 5, 78142-78149.

19 H. Zhong, Y. Liu, Z. F. Liu, Y. B. Jiang, F. Tan, G. M. Zeng, X. Z. Yuan, M. Yan, Q. Y. Niu and Y. S. Liang, Int. Biodeterior. Biodegrad., 2014, 94, 152-159.

20 Y. Ishigami, Y. Gama, H. Nagahora, M. Yamaguchi, H. Nakahara and T. Kamata, Chem. Lett., 1987, 763-766.

21 L. Chen and T. C. Kibbey, Langmuir, 2006, 22, 6874-6880.

22 S. Peng and M. L. Brusseau, Water Resour. Res., 2005, 41, 6874-6880.

23 M. J. Rosen, Surfactants and Interfacial Phenomena, John Wiley \& Sons, Hoboken, 3rd edn, 2004.

24 H. Zhong, Y. Jiang, G. Zeng, Z. Liu, L. Liu, Y. Liu, X. Yang, M. Lai and Y. He, J. Hazard. Mater., 2015, 285, 383-388.

25 NCBI, PubChem Compound Database, CID $=15600$, selected properties of $n$-decane, http://pubchem.ncbi.nlm. nih.gov/compound/15600, accessed May 27, 2015.

26 NCBI, PubChem Compound Database, CID $=8182$, selected properties of $n$-dodecane, http://pubchem.ncbi.nlm.nih. gov/compound/8182, accessed May 27, 2015.

27 NCBI, PubChem Compound Database, CID = 12389, selected properties of $n$-tetradecane, http://pubchem.ncbi. nlm.nih.gov/compound/12389, accessed May 27, 2015. 
28 NCBI, PubChem Compound Database, CID = 11006, selected properties of $n$-hexadecane, http://pubchem.ncbi. nlm.nih.gov/compound/11006, accessed May 8, 2015.

29 X. Z. Yuan, F. Y. Ren, G. M. Zeng, H. Zhong, H. Y. Fu, J. Liu and X. M. Xu, Appl. Microbiol. Biotechnol., 2007, 76, 1189-1198.

30 R. J. Zasoski, in Encyclopedia of Soil Science, ed. W. Chesworth, Springer, Netherlands, Dordrecht, 2008, pp. 841-845.
31 H. Zhong, G. M. Zeng, J. X. Liu, X. M. Xu, X. Z. Yuan, H. Y. Fu, G. H. Huang, Z. F. Liu and Y. Ding, Appl. Microbiol. Biotechnol., 2008, 79, 671-677.

32 D. A. Edwards, R. G. Luthy and Z. Liu, Environ. Sci. Technol., 1991, 25, 127-133.

33 D. A. Dzombak and F. M. M. Morel, Surface Complex Modeling, Hydrous Ferric Oxide, John Wiley \& Sons, New York, 1990.

34 R. J. Hunter, Zeta Potential in Colloid Science. Principles and Applications, Academic Press, New York, 1981. 